\title{
(BE)SCHIMPFEN im deutschen und polnischen Kontrast - Soziolinguistische und pragmatische Aspekte einer Sprechhandlung (am Beispiel der tierischen Schimpfnamen)
}

\section{Abusing/Insulting in German and Polish Contrast - Sociolinguistic and Pragmatic Aspects of the Speech Act (On the Example of Zoonotic Insults)}

Joanna Szczęk

\begin{abstract}
Abusing and insulting are verbal actions that are implemented in language in different ways. Specificity of abusing and linguistic means of expressing them results from many aspects of sociolinguistic and pragmatic nature. It is the concrete use of a given insult in the sitatuion context that launches its meaning in its entirety. The subject of the article are sociolinguistic and pragmatic aspects that influence usage and meaning of insults. Research material are zoonotic insults in German and Polish. The aim of the analysis is to indicate similarities and differences in the indicated scope.
\end{abstract}

\section{Keywords}

abuses, animals, zoonotic insults, sociolinguistics, pragmatics 


\section{Einführende Bemerkungen}

"Schimpfen ist die verbale Reaktion auf eine Frustration oder Versagung“ lautet ein bekanntes Zitat von Sigmund Freud (1922). Verbal wird es v. a. durch Gebrauch von Schimpfwörtern realisiert. Eine allgemeine Definition des Schimpfworts lautet „Beschimpfung, beleidigendes [derbes] Wort"1, das im Akt des SCHIMPFENS gebraucht wird, wobei dieser als „ein Angriffsakt durch abwertende, beleidigende Worte“ verstanden wird.

Im vorliegenden Beitrag wird auf soziolinguistische und pragmatische Mechanismen der Sprechhandlung (BE)SCHIMPFEN im Deutschen und im Polnischen eingegangen. Der Analyse liegt die Annahme zu Grunde, dass SCHIMPFEN ein Ausdruck der breit verstandenen Aggression ist (vgl. Aman 1973). Die empirische Grundlage für die geplante Studie sind deutsche und polnische Schimpfwörter, d. h. „Substantive, mit denen Personen anstatt mit ihren Namen oder Titel in abfälliger Weise angeredet oder benannt werden" (Seibicke 1996: 495), denen tierische Motive zu Grunde liegen und deren Schimpfpotenzial unterschiedlich ausgewertet werden kann. Ich konzentriere mich aber auf tierische Personennamen mit negativem Bewertungspotential. Das Ziel ist dabei, durch die Analyse der pragmatischen Aspekte der analysierten Schimpfwörter, Schlüsse in Bezug auf Ähnlichkeiten und Unterschiede in beiden Sprachen zu ziehen. Das Korpus der im Weiteren durchgeführten Analyse entstammt ausgewählten deutschsprachigen Wörterbüchern.

\section{Definitorisches}

Die Definition des Schimpfens umfasst zwei grundlegende Ebenen². Im Duden liest man dazu Folgendes:

1. a. ,seinem Unwillen, Ärger mit heftigen Worten [unbeherrscht] Ausdruck geben“;

b. ,jemanden schimpfend zurechtweisen, ausschimpfen';

2. a. ,(gehoben) jemanden herabsetzend, beleidigend als etwas bezeichnen';

b. ,(salopp spöttisch) etwas Bestimmtes zu sein vorgeben, sich als etwas nennen, bezeichnen'.

In den genannten Fällen wird die mit dem Verb bezeichnete Handlung im Bereich des Emotiven platziert. Der Definition kann man auch unterschiedliche Dimensionen der betreffenden Sprechhandlung entnehmen, die abhängig von der Situation unterschiedlich realisiert werden kann.

Im Polnischen findet man den Terminus obelga, den Grzegorczykowa (1991: 193) folgendermaßen definiert:

1 Vgl. http://www.duden.de/rechtschreibung/Schimpfwort, Zugriff am 26. 11. 2018.

2 Vgl. https://www.duden.de/rechtschreibung/schimpfen, Zugriff am 31. 8. 2018. 
[Beschimpfung - J. S.] ist immer ein Akt, der mit Worten realisiert wird und seitens des Täters ihr absichtlicher Bestandteil ein negatives Urteil ist, das sich auf den Empfänger bezieht (der beschimpfte Mensch), obwohl dieses Urteil manchmal nur zu einem Schimpfwort oder der Äußerung eines Schimpfortes werden kann. Beleidigen ist also ein gewisses verbales Verhalten, ein Ausdruck der negativen, verächtlichen Einstellung einer Gruppe von Menschen gegenüber anderen.

Außer schimpfen oder beschimpfen findet man auch andere Verben und Ausdrücke, die als Synonyme zu der ersten Definitionsebene gelten ${ }^{3}$ : vom Leder ziehen; (umgangssprachlich) blaffen, donnern, drauflosschimpfen, schnauzen, wettern; (abwertend) keifen; (umgangssprachlich abwertend) herumschreien; (österreichisch umgangssprachlich abwertend) keppeln; (emotional abwertend) zetern; (landschaftlich) kibbeln, schelten. Im Falle der zweiten, transitiven, Bedeutung werden folgende Synonyme im Deutschen genannt: anherrschen, ausschelten, ausschimpfen, die Meinung sagen, maßregeln, rügen, tadeln, zurechtweisen; (umgangssprachlich) anbrüllen, andonnern, anpfeifen, aufs Dach steigen, den Kopf waschen, die Leviten lesen, einen Rüffel geben, eine Standpauke / Strafpredigt halten, fertigmachen, heruntermachen, in den Senkel stellen, ins Gebet nehmen, rüffeln, rundmachen, sich vornehmen/vorknöpfen, zur Minna / Schnecke machen, zusammenstauchen; (schweizerisch umgangssprachlich) wüst sagen; (salopp) anscheißen, den Marsch blasen, einen Anschiss verpassen, herunterputzen; (derb) zur Sau machen, zusammenscheißen; (abwertend) anschreien; (umgangssprachlich abwertend) anschnauzen; (landschaftlich) abstauben, [aus]zanken.

Im Polnischen hat man es mit einer noch breiteren Vielfalt zu tun, wenn es um die Entsprechung des deutschen Verbs schimpfen geht. Fürs deutsche schimpfen findet man nämlich folgende Verben: pomostować, wymyślać (komuś), die folgendermaßen definiert werden: „krzyczeć na kogoś, używając obraźliwych słów ${ }^{6}$, wyzywać (kogoś) mit folgender Explizierung: ,używać wobec kogoś obelg i słów wulgarnych ${ }^{65}$, besztać, das wie folgt definiert wird: ,książk. słowami kierowanymi do kogoś wyrażać niezadowolenie z jego niewłaściwego postępowania w sposób zdecydowany i niezbyt delikatny', klać mit folgender Erklärung: ,wypowiadać wulgarne słowa lub przekleństwa' ${ }^{6}$ und przeklinać: ,wypowiadać wulgarne słowa lub przekleństwa ${ }^{67}$.

Vergleicht man die semantischen Charakteristika der Verben im Deutschen und im Polnischen, stellt man fest, dass alle genannten Verben einen gemeinsamen Nenner haben. Es handelt sich nämlich um Ausdruck von negativen Emotionen, dessen offensichtlichste Manifestation der Gebrauch von vulgären und beleidigenden Wörtern ist.

3 Vgl. https://www.duden.de/rechtschreibung/schimpfen, Zugriff am 31. 8. 2018.

4 Vgl. http://wsjp.pl/do_druku.php?id_hasla=32075\&id_znaczenia=3868555, Zugriff am 31. 8. 2018.

5 Vgl. http://wsjp.pl/do_druku.php?id_hasla=33914\&id_znaczenia=0, Zugriff am 31. 8. 2018.

6 Vgl. http://wsjp.pl/do_druku.php?id_hasla=25541\&cid_znaczenia=0, Zugriff am 31. 8. 2018.

7 Vgl. http://wsjp.pl/index.php?id_hasla=44361\&id_znaczenia=4683468\&l=21\&ind=0, Zugriff am 31. 8. 2018 . 


\section{Sprechhandlung (BE)SCHIMPFEN im Deutschen und im Polnischen}

(BE)SCHIMPFEN als eine Sprechhandlung kann sich v. a. gegen Menschen richten. Es kann aber auch gegen Tiere, Gegenstände oder Ereignisse gerichtet werden, wobei es aber hervorzuheben ist, dass diese in allen Fällen als Ausdruck negativer Emotionen gilt. In folgenden Ausführungen konzentriere ich mich auf das (BE)SCHIMPFEN gegen Menschen, da es der meiste Fall ist, anhand dessen auch eine breite Palette der pragmatischen und soziolinguistischen Aspekte thematisiert werden kann.

Schimpfen ist, psychologisch gesehen, „das Endglied einer dreigliedrigen Kausalkette [...] Aufs Äußerste reduziert, sieht diese Kette folgendermaßen aus:

Frustration (vereitelnde Ursache) $\rightarrow$ Affekt (Erregungszustand) $\rightarrow$ Aggression (Schimpfen).“ (vgl. Aman 1973)

Von Havryliv (2009: 69) wird (BE)SCHIMPFEN wie folgt ausgelegt: „präsens-indikative Äußerung des Sprechers an den anwesenden oder abwesenden Adressaten in Form einer Prädikation, die sich mit dem Ziel, Emotionen abzureagieren und/oder den Adressaten zu beleidigen, vollzieht und in der sowohl die absolute als auch die relative (okkasionelle) pejorative Lexik zum Einsatz kommt.” Demzufolge werden dem (BE) SCHIMPFEN zwei Funktionen zugeschrieben: eine emotive Funktion und eine pragmatische Funktion (vgl. ebd.). Das entspricht auch der Interpretation von Marten-Cleef (1991: 79), die SCHIMPFEN zu den EXPRESSIVA zählt und sie neben KLAGEN und KRITTELN als opponierende EXPRESSIVA mit Sprecher-Aversion betrachtet.

Wenn man die angeführten Definitionen und Versuche, die Bedeutung dieser Sprechhandlung auszulegen, vergleicht, stellt man fest, dass (BE)SCHIMPFEN v. a. in zwei Ausprägungen realisiert wird:

1. Abreagieren von Emotionen, wobei es sich um den intransitiven Gebrauch des die Sprechhandlung bezeichnenden Verbs handelt;

2. Abreagieren von Emotionen und Beleidigung des Adressaten.

Es muss auch ein Unterschied zwischen SCHIMPFEN und BESCHIMPFEN angemerkt werden. SCHIMPFEN erfolgt sowohl ohne Adressatenbezug, als auch mit Adressatenbezug. BESCHIMPFEN dagegen ist nur adressatenbezogen. Bei Marten-Clef (1991: 310) wird der Unterschied auch in der funktionalen Beschreibung beider Sprechhandlungen deutlich gemacht: Schimpfen habe nämlich die Funktion der emotionalen Entladung, Beschimpfen dagegen der der Kränkung eines Gegenübers. Dies bestätigt auch Sornig (1975: 160), indem er auf den perlokutiven Aspekt des Beschimpfens hinweist: „Der Hörer muß sich gekränkt fühlen [...], d. h. auch und vor allem (oder erst) der perlokutive Akt ist konstitutiv für das Sprechereignis Beleidigung.“

In der einschlägigen Literatur hebt man auch hervor, dass beim Beschimpfen drei Komponenten vorhanden sein müssen: Sprecher, Adressat und Mitteilung, wie es Opelt (1965: 15) expliziert: „Bei der Beschimpfung ist der Sprecher der heftig erregt Schimpfende, der Adressat der meist ebenso heftig erregte Beschimpfte; die Mitteilung besteht in der Äußerung des Schimpfwortes.“

Wenn man dann im Lichte dieser Ausführungen die beiden Sprechhandlungen vergleicht, erzielt man Folgendes: 
SCHIMPFEN $=$ Sprecher $(=$ Schimpfender $)+$ Mitteilung $(=$ Äußerung des Schimpfwortes) $)^{8}$

BESCHIMPFEN $=$ Sprecher $(=$ Schimpfender $)+$ Mitteilung $(=$ Äußerung des Schimpfwortes $)+$ Adressat $(=$ Beschimpfter $=$ Ursache des Beschimpfens $($ sein Verhalten, seine Eigenschaften, seine Handlungen usw.))

Daher kommt dem Adressaten eine doppelte Rolle zu: er wird beschimpft und stellt zugleich die Ursache des Beschimpfens dar.

\section{Funktionen des (BE)SCHIMPFENS}

Havryliv (2009: 69) legt die Funktionen des (BE)SCHIMPFENS im Lichte der definitorischen Ausführungen wir folgt aus: „präsens-indikative Äußerung des Sprechers an den anwesenden oder abwesenden Adressaten in Form einer Prädikation, die sich mit dem Ziel, Emotionen abzureagieren und/oder den Adressaten zu beleidigen, vollzieht und in der sowohl die absolute als auch die relative (okkasionelle) pejorative Lexik zum Einsatz kommt." Demzufolge werden dem BESCHIMPFEN zwei Funktionen zugeschrieben: eine emotive Funktion und eine pragmatische Funktion (ebd.).

Der Sprechhandlung BESCHIMPFEN liegen v. a. drei Funktionen zu Grunde (Schumann 1990: 262):

1. die Aggressionen des Beschimpfenden abzureagieren (sprecherorientiert), d.h. sich abreagieren des Beschimpfenden zum Lösen von Spannungen, affektiven Erregungen/Befriedigung von Ausdrucks-, Handlungsbedürfnissen/Steigerung des Selbstwertgefühls;

2. die Deklassierung des Beschimpften (adressatenorientiert); Herabsetzung des Beschimpften mit dem Ziel, ihn zu deprimieren/demütigen/kränken/reizen/ihm eine Niederlage zu bereiten.

3. An Dritten orientiert: Herabsetzung, Verunglimpfung des Beschimpften im Beisein anderer.

\section{Tierische Schimpfwörter und ihre Merkmale}

Ausdrucksmittel zur Ausführung der Sprechhandlung BESCHIMPFEN sind v. a. Schimpfwörter. Seibicke (1996: 495) erklärt das Wesen dieser sprachlichen Mittel wie folgt: „Schimpfwörter sind Substantive, mit denen Personen anstatt mit ihren Namen oder Titel in abfälliger Weise angeredet oder benannt werden”. Er schlägt auch vor, den Terminus Schimpfnamen zu gebrauchen, und gibt eine enge Definition des Schimpfwortes an: „Das Schimpfwort ist also nur ein spezielles Mittel für Beschimpfungen mit einem einzigen Wort." (ebd.)

8 Vgl. hierzu andere Bedeutungen der Sprechhandlung SCHIMPFEN u. a. bei Harras et al. (2007). 
Die thematischen Motive, die beim Schimpfen gebraucht werden, sind v. a. (vgl. Nübling, Vogel 2004: 19-33): Verunreinigung, Organe bzw. Produkte der Exkretion, Schmutz, Dreck, Abschaum, Mist, Scheiße, Arsch, pissen, Ekel oder Kotzen, Genitalien, sexuelle Handlungen, Promiskuität, Prostitution, Ehebruch, uneheliche Geburt, Virilität, Tiernamen, der religiöse Bereich, Krankheiten, geistige Unzulänglichkeiten. Seibicke (1996: 496) fasst es folgendermaßen zusammen:

Aus dem Inhalt der Schimpfreden ließe sich ein Katalog alles dessen zusammenstellen, was die Schimpfenden für verächtlich und verabscheuenswert halten. Das können körperliche Merkmale sein, geistige und moralische Defekte, barsches oder unsoziales Verhalten, Charaktereigenschaften [...], Verhaltensweisen [...], Unsauberkeit [...]. Insgesamt bilden sie einen negativen Tugendenspiegel.

Als Träger von bestimmten Eigenschaften und Wesen, die sich auf eine bestimmte Art und Weise verhalten, sind Tiere eine besonders gut geeignete Quelle, aus der man schöpft, um die anderen zu beleidigen und mit ihnen zu schimpfen. Dies ergibt sich aus der Tatsache, dass die Beziehungen zwischen Menschen und Tieren seit immer eng waren. Das bestätigt Lurker (1992: 752) folgendermaßen: „Tiere gelten seit eh und je als Träger von versteckten Wahrheiten über die Menschen selbst und der menschlichen Eigenschaften. Sie entlarven die menschlichen Schwächen und weisen deutlich auf deren Einschränkungen hin”. Daher ist es nicht verwunderlich, dass man zu diesem Bereich greift, um bestimmte Phänomene der Menschenwelt zu benennen. Es handelt sich dabei um „Metaphorisierungen, bei denen es zur Merkmalisolierungen kommt, deren Funktion pejorativ ist" (vgl. Zehan 2008: 374).

\section{Analyse des Materials}

In der Analyse konzentriere ich mich auf Personenschimpfwörter tierischer Provenienz im Deutschen und Polnischen. Es wird versucht, einen Katalog der funktionalen tierischen Charakteristika zu erstellen, die in den deutschen Personenschimpfwörtern verbalisiert werden. Das kann Schlüsse in Bezug auf deren pragmatischen Merkmale liefern.

Schon beim Vergleich der Struktur der trierischen Schimpfwörter im Deutschen und Polnischen lassen sich gewisse Unterschiede anmerken.

Der Struktur nach kann man folgende Typen von tierischen Personenschimpfwörtern unterscheiden (vgl. dazu Petershagen 2014):

1. einfache tierische Personenschimpfwörter, z. B.: im Deutschen: Schaf, Ochse, Esel u. a., im Polnischen: świnia, osiot u. a. Sie überwiegen im Polnischen.

2. tierische Personenschimpfwörter mit Eigenschaftswörtern in Form von Nominalphrasen mit adjektivischem Attribut meist vorangestellt, z. B.: im Deutschen: dumme Kuh, blöde Gans u. a.; im Polnischen: bura suka, zmokta kura u. a. Die Rolle, die den adjektivischen Attributen zukommt, ist die Hervorhebung der jeweiligen Eigenschaft und Verstärkung des Ausdrucks. Anzumerken ist jedoch auch, dass 
manche tierischen Personenschimpfwörter nur mit bestimmten Prädikaten vorkommen. Oft gibt es im Falle gleicher Tiere eine Differenzierung der Bedeutung durch den Zusatz eines Eigenschaftswortes, z. B.: fieses Schwein vs. armes Schwein;

3. ,neue' tierische Gattungsamen als Personenschimpfwörter, die nur im Deutschen vorkommen und Produkte der Wortbildung sind, z. B.: Dreckschwein, Sauhund, Angsthase, Lustmolch, Papiertiger, Hornochse, Mondkalb, Schafskopf, Meckerziege, Riesenross, bei denen durch den Zusatz von bestimmten Komponenten entweder eine Präzisierung oder Hyperbolisierung der Bedeutung erfolgt.

Die funktionalen und semantischen Charakteristika der tierischen Personenschimpfwörter im Deutschen und im Polnischen werden in der folgenden Tabelle zusammengestellt.

\begin{tabular}{|c|c|}
\hline Deutsch & Polnisch \\
\hline \multicolumn{2}{|c|}{ 1. Kollektiva - Menschengruppe } \\
\hline Affenstall, Hammelherde & $\begin{array}{l}\text { Szarańcza (dt. Schwarm), stado baranów (dt. Ham- } \\
\text { melherde) }\end{array}$ \\
\hline \multicolumn{2}{|c|}{ 2. Geschlecht } \\
\hline Männer, z. B.: Affenschwanz; & $\begin{array}{l}\text { Frauen, z. B.: bura suka (dt. graue Hündin); Männer, } \\
\text { z. B.: kundel (dt. Mischling); }\end{array}$ \\
\hline \multicolumn{2}{|c|}{ 3. Aussehen } \\
\hline $\begin{array}{l}\text { Größe, z. B.: Geißbock; ungepflegtes Aussehen, } \\
\text { Unsauberkeit, z. B.: für Frauen:Assel, Dreckschwal- } \\
\text { be; für Männer: Bärenhäuter, Dreckamsel, Ferkel, } \\
\text { Dreckferkel, Dreckhammel, Kanalratte, Kellerassel, } \\
\text { Mistfink, Sau, Schwein, Stänkerbock; Hässlichkeit, } \\
\text { z. B.: Affenfratze; für Frauen, z. B.: alte Eule, alte } \\
\text { Wachtel, grauslicher Uhu, Krähe, Kröte; seltsames } \\
\text { Aussehen, z. B.: Ameisenbär, Kauz, Vogel, komi- } \\
\text { scher Vogel, Paradiesvogel; für eine Frau: Huhn, } \\
\text { Krampfhenne; Fettleibigkeit, Rundlichkeit, z. B.: } \\
\text { für Frauen: Antilope, Kuh, Pferd; für Kinder: dicker } \\
\text { Mops; fette Sau, Mops, Nilpferd; Körperhaltung, z. } \\
\text { B.: steifer Bock; Körpermerkmale, darunter: langer } \\
\text { Hals: Giraffe; großer Busen: Milchkuh; helles Ge- } \\
\text { sicht: Molch; große Nase: Saurüssel; schmales Ge- } \\
\text { sicht und spitze Nase: Spitzmaus; unrasiertes Ge- } \\
\text { sicht: Stachelschwein; Armseligkeit, Schwachheit, } \\
\text { z. B.: arme Sau, begossener Pudel, Wurm, Floh, } \\
\text { graue Maus, halber Hahn, Kirchenmaus; äußere } \\
\text { Merkmale, z. B.: Brillenschlange; unnatürliche Kör- } \\
\text { perbräune, z. B.: Grillenhenne; }\end{array}$ & $\begin{array}{l}\text { Größe, z. B.: kobyła (dt. Stute); ungepflegtes Aus- } \\
\text { ssehen, Schlampigkeit, z. B.: flądra (dt. Flunder), } \\
\text { klępa (dt. Elchkuh), zmokła kura (dt. nasse Henne); } \\
\text { seltsames Aussehen, z. B.: koczkodan (dt. Küm- } \\
\text { merling); Fettleibigkeit, z. B.: maciora (dt. Sau), } \\
\text { ropucha (dt. Kröte); }\end{array}$ \\
\hline \multicolumn{2}{|c|}{ 4. Bewegungsart } \\
\hline \begin{tabular}{|l|} 
Elefant, Ente, lahme Ente, lascher Bock, Schnecke; \\
für Frauen: Wackelente;
\end{tabular} & boże cielę (dt. Gottes Kalb), ślimak (dt. Schnecke); \\
\hline \multicolumn{2}{|c|}{ 5. Alter } \\
\hline \begin{tabular}{|l|} 
dem Alter unangemessenes Verhalten, z. B.: alter \\
Esel, Kalb;
\end{tabular} & $\begin{array}{l}\text { junge Frau, z. B.: sikorka (dt. Meise); Mann, z. B.: } \\
\text { stary koń (dt. altes Pferd); }\end{array}$ \\
\hline \multicolumn{2}{|c|}{ 6. Eigenschaften } \\
\hline $\begin{array}{l}\text { Geschicktheit, z. B.: Aal, Aas; Habgier, z. B.: Aas- } \\
\text { geier; Dummheit, z. B.: für einen Mann, z. B.: Affe, } \\
\text { Affenschädel, alter Affe, blöder Hund, Esel, Frosch, } \\
\end{array}$ & $\begin{array}{l}\text { Dummheit, z. B.: baran (dt. Hammel), boże cie- } \\
\text { lę (dt. Gottes Kalb), cielak (dt. Kälbchen), dudek } \\
\text { (dt. Wiedehopf), gawron (dt. Saatkrähe), gęś (dt. }\end{array}$ \\
\hline
\end{tabular}


(BE)SCHIMPFEN im deutschen und polnischen Kontrast - Soziolinguistische und pragmatische ...

\begin{tabular}{|l|}
\hline \multicolumn{1}{|c|}{ Deutsch } \\
\hline Hirsch, Honigkuchenpferd, Kamel, Mastochse, \\
Maulesel, Mondkalb, Ochse, Pavian, Rhinozeros; \\
für Frauen: Amsel, blöde Gans, dumme Gans, dum- \\
me Kuh, Walross; \\
Pessimismus, z. B.: alte Unke; Ängstlichsein, z. B.: \\
Angsthase, Hase; \\
Faulheit, z. B.: Faultier; Gemeinheit, Niederträch- \\
tigkeit, z. B.: Bluthund, Hund räudiger Hund, \\
Sauhund; für Frauen, z. B.: Giftschlange, miese \\
Ratte; Grobheit, z. B.: Büffel, Hammel, Mistham- \\
mel, Tier, Werwolf; Falschheit, z. B.: Chamäleon, \\
Wetterhahn, Wolf im Schafspelz; für Frauen: fal- \\
sche Katze, Natter, Schlange; für Männer: falscher \\
Hund, krummer Hund; Erfahrungsmangel, Naivi- \\
tät, z. B.: Dachs, Gelbschnabel, heuriger Hase; für \\
Frauen: Gans, junger Dachs; Streitsucht, z. B.: für \\
Frauen: Drache, Dreckamsel, Gewitterziege, Haus- \\
drache; Geld und Habgier, z. B.: Finanzhyäne, Gei- \\
er, Hai, Hyäne;
\end{tabular}

Unzuverlässigkeit, z. B.: Fink; Schlauheit, z. B.: Fuchs, schlauer Fuchs, Ratte;

Eitelkeit, Hochnäsigkeit, z. B.: geleckter Affe, Gockel, Pfau; Sturheit, z. B.: Holzbock, sturer Bock; Gefühlskälte, Herzlosigkeit, z. B.: kalter Fisch;

Polnisch

Gans), gąka (dt. Gänslein), głupia koza (dt. dumme Ziege), osioł (dt. Esel); Grobheit, z. B.: bydlę (dt. Vieh), nieskrobane prosię (dt. ungeschältes Ferkel), prawdziwy niedźwiedź (dt. echter Bär); Stärke, z. B.: byk (dt. Bulle); Schwäche, z. B.: chrząszcz (dt. Käfer); Falschheit, z. B.: farbowany lis (dt. gefärbter Fuchs), gad (dt. Reptil), glista (dt. Spulwurm), padalec (dt. Blindschleiche), sójka (dt. Eichelhäher), szczur (dt. Rate), zdradziecki wąż (dt. verräterische Schlange), zjadliwa jaszczurka (dt. bissige Eidechse); Verwöhntsein, z. B.: francuski piesek (dt. französischer Hund); Hinterlist, z. B.: hiena (dt. Hyäne), kret (dt. Maulwurf), lis (dt. Fuchs), mądra ryba (dt. kluger Fisch); Boshaftigkeit, z. B.: istna wydra (dt. echter Fischotter), jadowity ozór (dt. bissige Zunge); Erfahrungsmangel, Naivität, z. B.: jeleń (dt. Hirsch), pospolita gąska (dt. durchschnittliche Gans), koza (dt. Ziege), pędrak (dt. Engerling), szczeniak (dt. Welpe); Wertlosigkeit, z. B.: klacz (dt. Stute), mała ryba (dt. kleiner Fisch), mucha (dt. Fliege), płotka (dt. Plötze), szarak (dt. Feldhase); Feigheit, z. B.: kot (dt. Katze), zając (dt. Hase); Verrücktsein, z. B.: małpa (dt. Affe); Aufgeblasensein, z. B.: nadymająca się żaba (dt. sich aufblasender Frosch); Hitzköpfigkeit, z. B.: odyniec (dt. Keiler); Stummheit, z. B.: ostryga (dt. Auster); Arbeitsamkeit, z. B.: wół roboczy (dt. Arbeitsochse); Niederträchtigkeit, z. B.: pies (dt. Hund); Skruppellosigkeit, z. B.: rekin (dt. Hai); Gefühlskälte, Herzlosigkeit, z. B.: zimna ryba (dt. kalter Fisch);

\section{Verhalten}

übermäßige sexuelle Gier, z. B.: für Männer: alter Bock, geiler Bock, Gockel, verliebter Gockel Hengst, Hurenbock, Specht; für Frauen: läufige Hündin; Ärgerursache, z. B.: blöde Kuh; Lautsein, z. B.: Brüllaffe; Diebstahl, z. B.: Elster, diebische Elster; Coolheit, z. B.: einsamer Wolf; Aufdringlichkeit, z. B.: Filzlaus, Laus, Qualle, Schmeißmücke; Frechheit, z. B.: für Frauen: freche Kröte, kesse Motte, kleine Kröte; Geschwätzigkeit, z. B.: Hendl; für Frauen: Lästerschwein; Rücksichtslosigkeit, z. B.: Pistensau, Pistenschwein; Unbeholfenheit, Ungeschicktheit, z. B.: Trampeltier; Trunksucht, z. B.: versoffenes Huhn;

Rowdytum, z. B.: bażant (dt. Fasan); Einsamkeit, z. B.: borsuk (dt. Dachs); Übermäßige sexuelle Gier, z. B.: byk (dt. Bulle), flądra (dt. Flunder), kogut (dt. Hahn), królik (dt. Kaninchen), pantera (dt. Panther); Ungezogenheit, z. B.: goryl (dt. Gorilla), orangutan (dt. Orang-Utan); Prostitution, z. B.: kawka (dt. Dohle), mewka (dt. Bordsteinschalbe), ptaszyna (dt. Vögelchen); Geschwätzigkeit, z. B.: kwoka (dt. Gockel), papuga (dt. Papagei); Grausamkeit, z. B.: lampart (dt. Leopard), ludzka hiena (dt. menschliche Hyäne), ludzki zwierz (dt. menschliches Tier), sęp (dt. Geier);

Verführerischsein, z. B.: małpeczka (dt. Äffchen); Religionsbezogenheit, z. B.: mysz kościelna (dt. Kirchenmaus); Schmarotzertum, z. B.: pasożyt (dt. Parasit), pijawka (dt. Blutegel); Faulheit, z. B.: niebieski ptak (dt. blauer Vogel);

\section{Fehlende Fähigkeiten}

Fehlende Schwimmfähigkeit, z. B.: bleierne Ente; Impotenz, z. B.: Kapaun;

\section{Berufe}

Baubranche: Baulöwe; Börse: Börsenhai; Polizis- Hausfrau, z. B.: kura domowa (dt. Haushenne); Poten: Bulle, Bullenschwein, Kettenhund, Polyp; Büro: lizisten: psy (dt. Hunde); 


\begin{tabular}{|l|l|}
\hline \multicolumn{1}{|c|}{ Deutsch } & Polnisch \\
\hline Bürohengst, Fliegenfänger; Jäger: Grünspecht; \\
Schreiner/Tischler: Holzwurm; Soldaten: Kam- \\
merbulle; Nonne: Pinguin; Lehrer: Tintenfuchs; \\
Prostituierte: Schwalbe, Strichvogel, Vögelchen, \\
Bordsteinschwalbe; Koch: Dunsthund; \\
\hline \multicolumn{2}{|c|}{ 10. Nationalitäten } \\
\hline & Wielbłąd (dt. Kamel); \\
\hline
\end{tabular}

Tab. 1 Zusammenstellung der funktionalen und semantischen Charakteristika von tierischen Schimpfwörtern im Deutschen und im Polnischen

Im Lichte der Zusammenstellung kann man schon einige Unterschiede quantitativer Art anmerken, denn auf den ersten Blick hat man es mit einer deutlichen Mehrheit der tierischen Personenschimpfwörter im Deutschen zu tun. Thematische Gruppen, die in beiden Sprachen mit besonders vielen Beispielen belegt sind, sind z. B. solche, die Aussehen des Menschen, dessen Eigenschaften und Verhalten thematisieren, wobei die Anzahl der Schimpfwörter in jedem Falle höher im Deutschen ist. Das kann verwundern, denn in Bezug auf Menschen scheinen die Erfahrungen der Sprachbenutzer aus beiden Sprachkulturen doch ähnlich, wenn nicht gleich zu sein. Im Falle der identischen Eigenschaften, die in beiden Sprachen in den tierischen Personenschimpfnamen thematisiert werden, handelt es sich um gleiche Tiere, was von der gleichen Wahrnehmung im jeweiligen Volk zeugt. Diese Tatsache verleitet wiederum zur Aufstellung der These, dass die in den Personenschimpfwörtern vorkommenden Tiere bestimmte hervorstechende und für sie typische Merkmale haben, die Grundlage für deren Übertragung auf Menschen bilden.

Anhand der oben angeführten Zusammenstellung kann man auch Charakteristika herausfiltern, die einzelnen Tieren in beiden Sprachkulturen nachgesagt werden, was ein Ausdruck der Wahrnehmung der Tiere im Konzept des sprachlichen Weltbildes ist. Das sprachliche Weltbild wird dabei folgendermaßen verstanden: „eine Ansammlung von Regelmäßigkeiten, die in den kategorialen grammatischen Beziehungen (die Flexion, die Wortbildung und die Syntax) und semantischen lexikalischen Strukturen enthalten sind, und welche die für eine Sprache eigentümliche Wahrnehmungsweise der Welt und allgemeines Verstehen der Weltgestalt, auch aller in der Welt vorhandenen hierarchischen Beziehungen und bestimmter Werte, die in einer Sprachgemeinschaft anerkannt werden, darstellt.“ (Tokarski 1993: 358, Übersetzung aus dem Polnischen - J. S.)

Die aus den tierischen Personenschimpfnamen ermittelten Merkmale der Tiere werden in der folgenden Tabelle zusammengestellt: 
(BE)SCHIMPFEN im deutschen und polnischen Kontrast - Soziolinguistische und pragmatische ...

\begin{tabular}{|c|c|c|}
\hline \multirow[t]{2}{*}{ Tier } & \multicolumn{2}{|c|}{ Charakteristika } \\
\hline & Deutsch & Polnisch \\
\hline Aal & geschickt; & \\
\hline Aas & gepflegt, gutaussehend, geschickt; & \\
\hline Affe & $\begin{array}{l}\text { ungepflegt, chaotisch, schmutzig, } \\
\text { schlampig, hässlich, dumm, eitel, } \\
\text { hochnäsig, laut; }\end{array}$ & verrückt, verführerisch; \\
\hline Ameisenbär & seltsam; & \\
\hline Amsel & $\begin{array}{l}\text { ungepflegt, schmutzig, schlampig, } \\
\text { dumm, streitsüchtig; }\end{array}$ & \\
\hline Antilope & dick, rundlich; & \\
\hline Assel & ungepflegt, schmutzig, schlampig; & \\
\hline Auster & & stumm; \\
\hline Bär & schmutzig, faul; & grob; \\
\hline Blindschleiche & & falsch; \\
\hline Blutegel & & schmarotzerhaft, parasitär; \\
\hline Bock & $\begin{array}{l}\text { groß, ungepflegt, schmutzig, schlam- } \\
\text { pig, steif, energielos, träge, stur, geil; }\end{array}$ & \\
\hline Büffel & grob; & stark; \\
\hline Bulle & & geil; \\
\hline Chamäleon & falsch; & \\
\hline Dachs & naiv, unreif; & einsam; \\
\hline Drache & streitsüchtig; & \\
\hline Eichelhäher & & falsch; \\
\hline Eidechse & & falsch; \\
\hline Elefant & langsam und schwerfällig; & \\
\hline Elster & diebisch; & \\
\hline Engerling & & unerfahren, naiv; \\
\hline Ente & lahm, wackelig; & \\
\hline Esel & alt, dumm; & dumm; \\
\hline Eule & hässlich; & \\
\hline Fasan & & rowdyhaft; \\
\hline Faultier & faul; & \\
\hline Feldhase & & wertlos, unbedeutend; \\
\hline Ferkel & ungepflegt, schmutzig, schlampig; & grob; \\
\hline Fink & $\begin{array}{l}\text { ungepflegt, schmutzig, schlampig, } \\
\text { unzuverlässig; }\end{array}$ & \\
\hline Fisch & gefühlskalt, herzlos; & $\begin{array}{l}\text { hinterlistig, wertlos, unbedeutend, ge- } \\
\text { fühlskalt, herzlos; }\end{array}$ \\
\hline Fischotter & & boshaft; \\
\hline Fliege & & wertlos, unbedeutend; \\
\hline Floh & schwach, armselig; & \\
\hline Flunder & & $\begin{array}{l}\text { ungepflegt, schlampig, aufgeblasen, } \\
\text { geil; }\end{array}$ \\
\hline Frosch & dumm; & \\
\hline Fuchs & schlau; & falsch, hinterlistig; \\
\hline Gans & dumm, naiv, unerfahren; & dumm, unerfahren naiv; \\
\hline Geier & habgierig; & grausam; \\
\hline
\end{tabular}


(BE)SCHIMPFEN im deutschen und polnischen Kontrast - Soziolinguistische und pragmatische ...

\begin{tabular}{|c|c|c|}
\hline \multirow[t]{2}{*}{ Tier } & \multicolumn{2}{|c|}{ Charakteristika } \\
\hline & Deutsch & Polnisch \\
\hline Giraffe & langhalsig; & \\
\hline Gockel & eitel, hochnäsig, geil; & geschwätzig; \\
\hline Gorilla & & ungezogen; \\
\hline Hahn & schwach, armselig, falsch; & geil; \\
\hline Hai & habgierig; & skrupellos; \\
\hline Hammel & $\begin{array}{l}\text { ungepflegt, schmutzig, schlampig, } \\
\text { grob; }\end{array}$ & dumm; \\
\hline Hase & ängstlich, naiv, unerfahren; & feige; \\
\hline Hengst & geil; & \\
\hline Henne & seltsam; & \\
\hline Heuschrecke & & in großen Mengen vorkommend; \\
\hline Hirsch & dumm; & unerfahren, naiv; \\
\hline Huhn, Hendl & seltsam, geschwätzig, trunksüchtig; & ungepflegt, niederträchtig; \\
\hline Hund & $\begin{array}{l}\text { gepflegt, gutaussehend, dumm, nie- } \\
\text { derträchtig, falsch; }\end{array}$ & verwöhnt; \\
\hline Hündin & geil; & gemein; \\
\hline Hyäne & habgierig; & hinterlistig, grausam; \\
\hline Käfer & & schwach; \\
\hline Kalb & & langsam, dumm; \\
\hline Kamel, Trampeltier & dumm, ungeschickt; & \\
\hline Kaninchen & & geil; \\
\hline Kapaun & impotent; & \\
\hline Kauz & seltsam; & \\
\hline Katze & falsch; & feige; \\
\hline Keiler & & hitzköpfig; \\
\hline Krähe & hässlich; & \\
\hline Kröte & hässlich, frech; & dick, fettleibig, rund; \\
\hline Kuh & $\begin{array}{l}\text { dick, rundlich, großer Busen, dumm, } \\
\text { Ärger erregend; }\end{array}$ & \\
\hline Laus & aufdringlich; & \\
\hline Leopard & & grausam; \\
\hline Löwe & & \\
\hline Maulwurf & & hinterlistig; \\
\hline Maus & $\begin{array}{l}\text { schmales Gesicht, spitze Nase, } \\
\text { schwach, armselig; }\end{array}$ & religionsbezogen; \\
\hline Meerkatze & & seltsam; \\
\hline Mops & dick, rundlich; & \\
\hline Motte & frech; & \\
\hline Mücke & aufdringlich; & \\
\hline Natter & falsch; & \\
\hline Nilpferd & dick, rundlich; & \\
\hline Ochse & dumm; & arbeitsam; \\
\hline Orang-Utan & & ungezogen; \\
\hline Panther & & geil; \\
\hline Papagei & & geschwätzig; \\
\hline
\end{tabular}


(BE)SCHIMPFEN im deutschen und polnischen Kontrast - Soziolinguistische und pragmatische ...

\begin{tabular}{|c|c|c|}
\hline \multirow[t]{2}{*}{ Tier } & \multicolumn{2}{|c|}{ Charakteristika } \\
\hline & Deutsch & Polnisch \\
\hline Parasit & & schmarotzerhaft, parasitär; \\
\hline Pavian & dumm; & \\
\hline Pfau & eitel, hochnäsig; & \\
\hline Pferd & dick, rundlich; & \\
\hline Plötze & & wertlos, unbedeutend; \\
\hline Pudel & schwach, armselig; & \\
\hline Qualle & aufdringlich; & \\
\hline Ratte & $\begin{array}{l}\text { ungepflegt, schmutzig, schlampig, } \\
\text { niederträchtig, gemein, schlau; }\end{array}$ & falsch; \\
\hline Regenwurm & & falsch; \\
\hline Reptil & & falsch; \\
\hline Rhinozeros, Nashorn & dumm; & \\
\hline Saatkrähe & & dumm; \\
\hline Sau & $\begin{array}{l}\text { ungepflegt, schmutzig, schlampig, } \\
\text { dick, rundlich, schwach, armselig, } \\
\text { rücksichtslos; }\end{array}$ & dick, fettleibig, rund; \\
\hline Schlange & $\begin{array}{l}\text { mit Brille, niederträchtig, gemein, } \\
\text { falsch; }\end{array}$ & falsch; \\
\hline Schnecke & langsam, energielos; & langsam; \\
\hline Schwalbe & schmutzig, schlampig; & \\
\hline Schwein & $\begin{array}{l}\text { ungepflegt, schmutzig, schlampig, } \\
\text { geschwätzig, rücksichtslos; }\end{array}$ & $\begin{array}{l}\text { ungepflegt, schmutzig, schlampig, ge- } \\
\text { schwätzig, rücksichtslos; }\end{array}$ \\
\hline Specht & geil; & \\
\hline Stachelschwein & unrasiert; & \\
\hline Stute & & groß, wertlos, unbedeutend; \\
\hline Tier & grob; & grausam; \\
\hline Uhu & hässlich; & \\
\hline Unke & pessimistisch; & \\
\hline Vieh & & grob; \\
\hline Vogel & seltsam; & faul; \\
\hline Wachtel & hässlich; & \\
\hline Walross & dumm; & \\
\hline Welpe & & unerfahren, naiv; \\
\hline Werwolf & grob, brutal; & \\
\hline Wiedehopf & & dumm; \\
\hline Wolf & falsch, hinterlistig, cool; & \\
\hline Wurm & schwach, armselig; & \\
\hline Ziege & streitsüchtig; & dumm, unerfahren, naiv; \\
\hline
\end{tabular}

Tab. 2 Merkmale der Tiere in den tierischen Personenschimpfwörtern im Deutschen und im Polnischen

Auch in diesem Falle sind einige Asymmetrien beobachtbar, die in der Zuschreibung von Eigenschaften einzelnen Tieren erkennbar sind. Im Deutschen werden folgende Tierbezeichnungen in den tierischen Personenschimpfnamen nicht gebraucht: Blindschleiche, Blutegel, Bulle, Eichelhäher, Eidechse, Engerling, Feldhase, Fischotter, Fliege, Gorilla, 
Heuschrecke, Käfer, Kalb, Kaninchen, Keiler, Leopard, Löwe, Maulwurf, Meerkatze, Orang-Utan, Panther, Papagei, Parasit, Pinguin, Plötze, Regenwurm, Reptil, Saatkrähe, Stute, Vieh, Welpe, Wiedehopf. Im Polnischen dagegen sind es: Aal, Aas, Ameisenbär, Amsel, Antilope, Assel, Bock, Chamäleon, Drache, Elefant, Elster, Eule, Ente, Faultier, Fink, Floh, Giraffe, Hengst, Henne, Kamel, Kapaun, Kauz, Krähe, Laus, Mops, Motte, Mücke, Natter, Nilpferd, Pavian, Pfau, Pferd, Pudel, Qualle, Nashorn, Specht, Stachelschwein, Uhu, Unke, Werwolf, Wolf, Wurm. Wie man sieht, handelt es sich dabei oft um exotische, selten vorkommende oder kaum wahrnehmbare Tiere.

Manchen Tieren werden mehrere differente Eigenschaften nachgesagt, wie z. B. dem Fisch, der Flunder, dem Huhn oder dem Schwein. Im Falle der Tierbezeichnungen, die in beiden Sprachen Komponenten der Personenschimpfwörter sind, sind bezüglich der ihnen zugeschriebenen Charakteristika auch kleine Divergenzen anzumerken. Diese betreffen die Wahrnehmung folgender Tiere: Affe, dem im Polnischen zusätzlich das Merkmal [verführerisch] zugeschrieben wird, Dachs, der im Deutschen eher naiv, unreif und im Polnischen einsam ist, Gockel, die im Deutschen als Symbol der Eitelkeit, Hochnäsigkeit und Geilheit gilt, während sie im Polnischen mit Geschwätzigkeit assoziiert wird, Hahn, dem im Deutschen Schwachheit, Armseligkeit und Falschheit nachgesagt wird, und im Polnischen als Symbol der Geilheit wahrgenommen wird, Ziege, die Träger der Streitsucht im Deutschen und der Naivität und Dummheit im Polnischen ist.

\section{Schlussfolgerungen}

Die Zusammenstellung der Personenschimpfwörter tierischer Provenienz lässt einige Folgerungen in Bezug auf deren pragma- und soziolinguistische Reflexe im deutsch-polnischen Vergleich ziehen.

Im Deutschen überwiegen tierische Personenschimpfwörter in Form von Komposita. In diesen bilden Tierbezeichnungen am häufigsten die Basis, Bestimmungswörter sind dagegen oft Nomina, die bestimmte Eigenschaften beim richtigen Namen nennen, z. B.: Dreckamsel, Lästerschwein, Stänkerbock. Dieselbe Rolle kommt auch den Adjektiven zu, die Komponenten der deutschen Personenschimpfwörter in Form von Nominalphrasen sind, z. B.: fette Sau, alte Eule, dicker Mops, dumme Kuh. Mit derselben Funktion der Adjektive hat man es auch im Polnischen zu tun, wo tierische Personenschimpfwörter in Form von Komposita kaum vorhanden sind und eher die Nominalphrasen mit adjektivalen Attributen überwiegen, z. B.: gtupia koza, farbowany lis, zjadliwa jaszczurka, nadymajaca sie żaba. Solchen Elementen, die stabile Komponenten der tierischen Personenschimpfwörter (Bestimmungswörter, Adjektive) sind, kommt eine pragmatische Rolle zu. Sie sollen nämlich zum richtigen Verständnis der Bedeutung verhelfen, indem sie negative Eigenschaften direkt nennen und keinen Platz für Interpretation lassen.

Der Vergleich der Eigenschaften, die den Tieren nachgesagt werden, lässt die These aufstellen, dass in beiden Sprachkulturen nicht nur fast gleiche Tiere, sondern auch deren fast gleiche Eigenschaften zum Gegenstand der menschlichen Sprechhandlung (BE) SCHIMPFEN werden. Das ist auch nicht verwunderlich, denn die beiden Völker sind 
benachbart, was auch gewisse Ähnlichkeiten in deren Lebensweise und Wahrnehmung der Welt vermuten lässt.

Zum Schluss sei auf eine wichtige Tatsache hingewiesen. Die Wahrnehmung der Tiere und Übertragung der Eigenschaften, die denen von uns, Deutschen und Polen, zugeschrieben werden, ist eine Konvention, die es in den meisten Fällen kaum etwas mit der Wirklichkeit zu tun hat. Dies fasst Dröscher (1988: 9) folgendermaßen zusammen:

Wenn [...] nächstens beschimpft werden als ,dummer Esel', ,dreckiges Schwein', ,flausiger Windhund“ oder ,tapsiger Elefant im Porzellanladen', seien Sie stolz! Denn mit Blick auf das Tierreich entpuppt sich der Tadel als hohes Lob. [...] Über 250 Sprichwörter mit Mensch-Tier-Vergleichen habe ich mit meiner vierzehnjährigen Erfahrung auf dem Gebiet wissenschaftlicher Verhaltensforschung auf ihren Wahrheitsgehalt untersucht. Das Resultat ist niederschmetternd. Fast drei Viertel der Redensarten stellen die Tatsachen auf den Kopf.

\section{Literaturverzeichnis}

Aman, Reinhold (1973): Bayrisch-österreichisches Schimpfwörterbuch. München.

Dröscher, Vitus B. (1988): Sie turteln wie die Tauben. Hamburg.

Grzegorczykowa, Renata (1991): „Obelga jako akt mowy“. In: Poradnik Językowy 5-6, S. 193-200.

Freud, Sigmund (1922): Totem und Tabu. Leipzig.

Harras, Gisela et al. (2007): Handbuch deutscher Kommunikationsverben. Berlin / New York.

Havryliv, Oksana (2009): Verbale Aggression: Formen und Funktionen am Beispiel des Wienerischen. Frankfurt a. M.

Lurker, Manfred (1992): Wörterbuch der Symbolik. Stuttgart.

Marten-Cleef, Susanne (1991): Gefühle ausdrücken. Die expressiven Sprechakte. Göppingen.

Nübling, Damaris, Marianne Vogel (2004): „Fluchen und Schimpfen kontrastiv. Zur sexuellen, krankheitsbasierten, skatologischen und religiösen Fluch- und Schimpfwortprototypik im Niederländischen, Deutschen und Schwedischen“. Germanistische Mitteilungen 59, S. 19-33.

Opelt, Ilona (1965): Die lateinischen Schimpfwörter und verwandte sprachliche Erscheinungen. Eine Typologie. Heidelberg.

Petershagen, Henning (2014): Beleidigungen und Kosenamen auf tierischer Basis, 5. 4. 2014. Zugriff am 4. 1. 2019. https://www.tagblatt.de/Nachrichten/Beleidigungen-und-Kosenamen-auftierischer-Basis-87707.html.

Pfeiffer, Herbert (1996): Das große Schimpfwörterbuch. Frankfurt a. M.

Schumann, Hanna (1990): Sprecherabsicht: Beschimpfung, In: Zeitschrift für Phonetik, Sprachwissenschaft und Kommunikationsforschung 43, S. 259-281.

Seibicke, Wilfried (1996): Nachwort. In: Das große Schimpfwörterbuch, hrsg. v. Herbert Pfeiffer. Frankfurt a. M.

Skawiński, Jacek, Artur Tworek (2003): Tiernamen als Schimpfwörter im Polnischen und im Deutschen. In: Beiträge der Europäischen Slavistischen Linguistik (POLYSLAV), 6, S. 166-170.

Sornig, Karl (1975): Beschimpfungen. In: Grazer linguistische Studien, Bd.1: Sprache und Gesellschaft, Graz, S. 150-167.

Tokarski, Ryszard (1993): Słownictwo jako interpretacja świata. In: Encyklopedia kultury polskiej XX wieku, t. 2: Współczesny język polski, hrsg. v. Jerzy Bartmiński, Wrocław, S. 335-363. 
Zehan, Rodica Liana (2008): Schimpfwörter gebildet aus deutschen Tiernamen. In: Research Journal of Agricultural Science Vol. 40, No. 3, S. 373-378.

Dr hab. Joanna Szczęk / joanna.szczek@uwr.edu.pl

Universität Wrocław, Institut für germanische Philologie,

PI. Nankiera 15 b, 50-140 Wrocław, Polska

This work can be used in accordance with the Creative Commons BY-SA 4.0 International license terms and conditions (https://creativecommons.org/licenses/by-sa/4.0/legalcode). This does not apply to works or elements (such as image or photographs) that are used in the work under a contractual license or exception or limitation to relevant rights 
